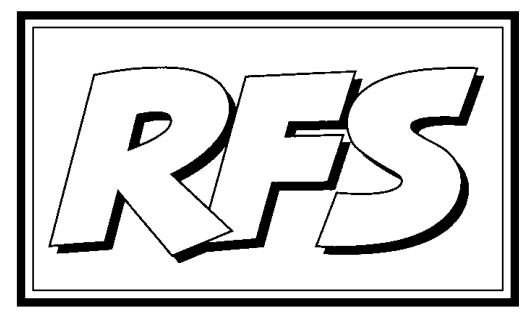

Revista de Fomento Social, 58 (2003), 105-134

\title{
El seguro de dependencia: Una nueva perspectiva del envejecimiento ${ }^{1}$
}

J avier MOYA HUERTOS

“No existe en los seres vivos un proceso más natural que el envejecimiento, y sólo se conoce un ser vivo que lucha contra él denodada e infructuosamente, el Hombre".

\section{Introducción}

Mediante este artículo se pretende realizar una aproximación a los distintos enfoques que en el Primer Mundo se están ofreciendo al continuo crecimiento de las situaciones de dependencia de su población más anciana.

1 Conferencia impartida el 12 de diciembre de 2001 con motivo de la clausura del II Master en Dirección de Centros Gerontológicos organizado por ETEA en Córdoba.

* Jefe del Servicio de Inspección de la Delegación Provincial de la Consejería de Asuntos Sociales en Córdoba. 
Y hablaré de los países del eufemísticamente llamado "mundo desarrollado" porque, si bien es cierto que no se trata ésta de una cuestión privativa de los países económicamente desarrollados, tampoco lo es menos que son éstos los únicos que, por su solvencia económica, se encuentran en condiciones de ofrecerle una solución. Surgen aquí importantes planteamientos éticos que no pueden ser ajenos al contenido de este trabajo ni a la línea editorial de esta publicación. ¿Es justo luchar por la prolongación de la vida de los ciudadanos del Primer Mundo cuando la inmensa mayoría de los habitantes del Tercer Mundo claman tan sólo por saciar su hambre? ¿No son estos unos planteamientos propios de la "ética del bienestar"? ¿Es un lujo tratar esta cuestión en un mundo donde reina la injusticia?

Por otra parte, dependencia y envejecimiento son conceptos que suelen asemejarse pero que en absoluto pueden considerarse como sinónimos. De hecho, se puedellegar a anciano sin padecer situaciones de dependencia que requieran del auxilio de otra persona para realizar las actividades básicas de la vida diaria (comer, vestirse, asearse, moverse), al igual que se puede ser muy dependiente a una edad muy temprana (Ej. tetraplejias producidas por accidentes de tráfico). Son estas situaciones de dependencia (postración en cama, alimentación mediante sonda nasogástrica, necesidad de cuidados paliativos, ingresos en residencias de asistidos, atención domiciliaria continua) en personas ancianas que, sin padecer una enfermedad merecedora de ingreso hospitalario, requieren una atención continuada muy especializada, y por tanto costosa, las que constituyen el objeto de este trabajo. El hecho de que en nuestro país aún no se haya comenzado una profunda reflexión sobre esta cuestión no significa, en modo alguno, que el problema carezca de relevancia. Confío en que este análisis de la situación, así como la aproximación a las soluciones que al mismo han ofrecido los países de nuestro entorno, contribuyan a generar una conciencia de la gravedad del problema $y$, en la medida de lo posible, permita atisbar algunas posibles soluciones.

El nuevo siglo nos trae nuevos problemas, y uno de ellos, que nos acecha de forma silenciosa, es el de cómo debe afrontar la sociedad las eventuales situaciones de dependencia de sus cada día más longevos miembros. El progreso técnicocientífico alcanzado por la humanidad nos puede y debe aportar nuevas soluciones, pero al dilema ético que subyace bajo esta cuestión sólo se le puede dar respuesta desde lo profundo de la conciencia humana. 


\section{El envejecimiento como problema}

El envejecimiento de las sociedades económicamente avanzadas se plasma en un espectacular crecimiento de la longevidad de sus individuos hasta límites nunca conocidos en la historia de la humanidad. Esto, que en sí mismo constituye uno de los mayores y más silenciosos logros de la especie humana, conlleva el surgimiento de nuevos problemas; uno de ellos lo constituye la dependencia, y su atención nos reclama soluciones eficaces y novedosas.

Sobre este asunto existen multitud de informes y estudios que revelan el preocupante y progresivo envejecimiento de la población mundial. Los datos son sobradamente conocidos por cualquier persona que se acerque al mundo de la atención a las personas mayores y, por ese motivo, realizaréuna muy simple exposición, a título de mero recordatorio, de las cifras que a nivel global se manejan sobre esta cuestión.

- La población mundial de más de 65 años se incrementa en 750.000 personas por mes, lo que significa que cada año, en nuestro planeta, nueve millones de personas alcanzan dicha edad.

- En el año 2025 habrá en el mundo 800 millones de personas mayores de 65 años, encontrándose $2 / 3$ de esta cifra en países en desarrollo y siendo la mayoría de ellas mujeres.

- Sólo en China habrá 274.000 .000 de personas mayores de 60 años, cifra muy superior a la población estadounidense actual.

- En los próximos 30 años se esperan incrementos en la población más anciana superiores al 300\% en muchos países en desarrollo, especialmente en América latina y Asia.

- En el año 1950 había sólo 200 personas de más 100 años en Francia, en el 2000 ya había 8.500, y para el 2050 se espera que la población centenaria francesa esta cifra alcance a 150.000 personas; lo que constituye un incremento del $750 \%$ en 100 años.

Paralelamente al vertiginoso crecimiento, en términos históricos, de la esperanza de vida del hombre, el núcleo familiar en el que el individuo se inserta ha sufrido sustanciales modificaciones que inciden directamente en las situaciones de dependencia: incorporación de la mujer al trabajo, alejamiento del modelo convencional de pareja casada con niños hacia formas domésticas más pequeñas y cambiantes: parejas sin hijos, personas solteras 
sin hijos, familias monoparentales, menor número de hijos, etc.

Antes de seguir profundizando en el tema que hoy nos ocupa, debemos definir el concepto de dependencia. Para ello podemos acudir a diversas fuentes, entre las que merecen destacarse la Organización Mundial de la Salud y el Consejo de Europa. La OMS define la dependencia como "las limitaciones de orden físico o mental que requieren la ayuda de otras personas". Por su parte, el Consejo de Europa definía en 1998 como dependiente a "la persona que, por razones ligadas a la falta o la pérdida de capacidad física, psíquica o intelectual, tiene necesidad de una asistencia o ayuda importante para la realización de las actividades de la vida diaria".

Quedémonos con esta simple idea: la dependencia supone una limitación de la autonomía personal de un nivel tal, que hace necesaria la participación de otra para realizar actividades elementales de la vida (comer, vestirse, asearse, moverse).

La Comisión Europea, en su informe de 1998 “La dependencia a largo plazo de las Personas Mayores" demuestra que la dependencia aumenta exponencialmente con la edad, afirmando que el $10 \%$ de las personas de 75 o más años son dependientes, y un $25 \%$ parcialmente dependientes.

En cualquier caso, los conceptos de vejez y dependencia, pese a estar relacionados entre sí, no son en absoluto sinónimos. De hecho, y aun admitiendo que las proyecciones demográficas expuestas fuesen totalmente correctas, ello no quiere decir que se vaya a producir un aumento en idéntica o similar proporción delas personas mayores dependientes. Esta circunstancia dependerá directamente de cómo evolucione la prevalencia de la incapacidad funcional en el futuro. Admitir que ésta no sufrirá alteración es lo que alimenta las visiones tan pesimistas y alarmantes del envejecimiento demográfico, pero estas tesis no tienen por qué ser ciertas y, sobre todo, debemos de ser conscientes de que aún estamos a tiempo de evitar que lo sean 0 , cuando menos, de minorar la gravedad de sus consecuencias.

En apoyo de las tesis menos alarmistas, y a modo de ejemplo, merece citarse el hecho de que un estudio realizado por científicos norteamericanos $^{2}$ ha demostrado que la tasa de prevalencia ${ }^{3}$ de la dependencia de EE.UU.

2 JaCobzone y otros, (1999)

3 Se entiende por tasa de prevalencia de la dependencia la evolución de la incidencia de la dependencia en una sociedad durante un determinado período de tiempo. 
seha reducido en 1,3 puntos porcentuales entre los años 1993 y 1998, en parte debido a cuestiones tan elementales como la mejor calidad de la alimentación y la higiene que han recibido las generaciones de americanos que ahora llegan a pertenecer a la llamada "cuarta edad", los mayores de 80 años, y en parte a los avances científicos y técnicos que han venido a minorar la incidencia de la dependencia, permitiendo prolongar la autonomía personal por más tiempo.

De hecho, Jacobzone llega a clasificar a varios países en tres grupos en función de la evolución registrada en sus tasas de dependencia. Su clasificación es la siguiente:

- Países en los que se aprecian reducciones significativas de sus tasas de dependencia: EE.UU., Alemania, Francia, Japón y, aunque con menor intensidad, Suecia.

- Países en los que no existen reducciones o éstas son moderadas: Australia y Reino Unido.

- Países en los que no se aprecia ninguna tendencia clara: Canadá y Holanda.

(Lamentablemente, en España no se dispone de información estadística fiable sobre la evolución de un grupo significativo de individuos en el tiempo y sobre la incidencia en ellos de las situaciones de dependencia, sino de información sobre población dependiente limitada a un momento en concreto, lo que impide realizar un estudio tan riguroso como el elaborado por los científicos americanos).

En cualquier caso, los límites del conocimiento humano no pueden dejar de evolucionar ante el fenómeno del envejecimiento que, precisamente por ser tan novedoso en términos históricos, (recordemos que la esperanza de vida media de un español en 1900 era de 35 años), será objeto de multitud de investigaciones científicas que redundarán en una mejor calidad de vida en la vejez. De hecho, los últimos avances en biología molecular ya comienzan a desvelar las claves genéticas del envejecimiento físico humano.

Sobre lo que no hay duda es que el natural y progresivo deterioro físico y mental de la persona, unido a la eventual disminución de su capacidad de valerse por sí mismo, hacen preciso el desarrollo de sistemas capaces de permitir suplir sus carencias. El interés que la Sociedad y las Autoridades están mostrando por esta problemática está desembocando ya en algunas 
propuestas originales que tienen su origen en el mundo del seguro, y que constituyen el objeto de este trabajo.

En definitiva, las causas últimas del nacimiento de la necesidad de ofrecer cobertura a los supuestos de dependencia son dos:

- el escenario demográfico, presente y futuro, y

- el cambio de estructura de la familia.

La Comisión de la Unión Europea, en un informe de 21 de mayo de 1999 titulado "Hacia una Europa para to das las edades", formulaba varias observaciones que merecen ser destacadas:

- Sólo entre 1960 y 1995, la esperanza media de vida para los ciudadanos de la UE aumentó en 8 años para los hombres y en 7 para las mujeres. Este aumento de la esperanza de vida, unido al descenso de la fecundidad por debajo del nivel de sustitución, hará que el envejecimiento demográfico constituya un fenómeno no sólo evidente, sino también inquietante en el siglo que ahora comienza.

- Por otra parte, estudios demográficos aseguran que en los próximos 25 años el porcentaje de crecimiento de la población en edad activa con respecto a la actual experimentará un incremento del $46 \%$ frente al $88 \%$ en que se espera que crezca la población mayor de 65 años. Esta situación nos conduce de manera inexorable hacia el objetivo de este trabajo: el problema de la financiación de las necesidades de atención de nuestros mayores y, en concreto, de los mayores dependientes. ¿Quiénes y cómo habrán de hacer frente a este enorme gasto? ¿Responderán los actuales sistemas de protección social ante este ingente incremento de las prestaciones?

En España, el costeanual dela atención a las perso nas dependientes (entre 950.000 y 1.270 .000 en 1998) se sitúa entre los $7.212 .000 €(1,2$ billones de pesetas) según fuentes de la Patronal del Seguro (Unespa), y los 18.030.000 $€$ (tres billones de pesetas) según otras fuentes consultadas. En cualquier caso, y pese a la disparidad de las cantidades manejadas, la cifra es escalofriante, y el problema, una vez determinada la necesidad de afrontarlo, asunto éste que afortunadamente nadie parece cuestionar, se centra en torno a su financiación. 


\section{Algunas aclaraciones previas}

Si bien las posibles alternativas de financiación constituyen un problema en sí mismo, no es menos cierto que para conocer el montante global de la cifra que habrá que financiar y, lo que es más importante, para definir el modelo de seguro de dependencia por el que opta nuestro país, habrá antes que determinar cuestiones como:

- el alcance de la cobertura de este seguro,

- el carácter público, privado o mixto del mismo,

- un mecanismo ágil que permita la clara distinción de lo que es un supuesto de dependencia de lo que lo es de enfermedad y

- la determinación del tipo de prestación que deberá ofrecer la compañía aseguradora (pago de cantidad o prestación de servicio).

Llegados a este punto, conviene comentar algunas de estas cuestiones antes de adentrarnos en el contenido del seguro de dependencia. Comencemos, pues, realizando algunas aclaraciones previas.

- No es en absoluto baladí la cuestión de decidir si esta modalidad de seguro habrá de ser público, privado o mixto, es decir, si debe incluirse o no la atención a las personas dependientes dentro de las coberturas propias de la Seguridad Social, si debe excluirse de ella o si debe optarse por un sistema intermedio.

A la vista de la actual regulación y funcionamiento de la asistencia sanitaria en nuestro país, muchos ciudadanos han suscrito un Seguro de Asistencia Médica a la vez que mantienen sus cotizaciones a la Seguridad Social. Nos encontramos pues un supuesto de doble pago de la misma prestación, dado que el servicio prestado no difiere sustancialmente. En este sentido, se están perdiendo recursos económicos que podrían dedicarse, por ejemplo, para la creación de costosas infraestructuras como las destinadas a la atención de personas dependientes: residencias de mayores asistidos, residencias de gravemente afectados, centros sociosanitarios). De esta forma, si nos hallásemos ante una cobertura complementaria a la ofrecida por el sistema público habríamos optimizado los recursos disponibles a la vez que habríamos ampliado la protección ofrecida. Esta opción, más racional, es la que ha adoptado, por ejemplo, el gobierno federal alemán y que expondremos con posterioridad. Por ello, y concluyendo, en el caso de optar por un 
modelo mixto, definido por caracteres privados y públicos, habrá de tenerse especial cuidado en que las coberturas sean complementarias, para de esta forma, aunar esfuerzos, sumar coberturas y aprovechar todos los recursos disponibles para posibilitar una mejor atención a las personas dependientes.

- Otro concepto que conviene aclarar antes de profundizar más en el asunto que nos ocupa, es la distinción de los conceptos de atención a la dependencia y de atención a la enfermedad. Ambas situaciones disponen de una modalidad de seguro específica, y su cobertura debe ser absolutamente independiente. Una persona que (a partir de la edad que la póliza establezca) deba recibir cuidados derivados de su situación de dependencia, recibirá éstos a cargo de su seguro de dependencia, pero si el supuesto consiste en que el asegurado debe recibir atención médica con motivo de enfermedad o accidente, con independencia de cuál sea su edad, estos cuidados deberán prestarse a cargo de su seguro de enfermedad. Todas las regulaciones existentes del seguro de dependencia establecen mecanismos para distinguir una situación de otra (tribunales de valoración multidisciplinares), dado que la confusión de situaciones tan íntimamente vinculadas en edades avanzadas puede producir evidentes perjuicios en primer lugar para los asegurados, pero también para el sistema que garantice la prestación (ya sean la Seguridad Social, una compañía aseguradora o ambas, si se trata de un seguro de tipo mixto) que para sanear su cuenta de resultados se vería obligada a incrementar el importe de las aportaciones de los asegurados y, en consecuencia, se acabaría cuestionando la propia viabilidad de esta modalidad de cobertura del riesgo de dependencia.

- No menos importante que la anterior es la necesaria distinción entre los conceptos de atención a la dependencia y atención socio sanitaria. Si la dependencia no es una cuestión privativa de los mayores, tampoco la atención sociosanitaria lo es de la dependencia. La atención sociosanitaria debe de ser una pieza más en el complejo entramado de prestaciones que ha de integrar un completo sistema de cobertura a la dependencia, el cual debe comprender, además de recursos cuasi hospitalarios, prestaciones económicas y técnicas, así como medidas fiscales, tanto a favor de las personas dependientes como de sus cuidadores informales, y cualesquiera otras medidas que favorezcan la compatibilidad del desarrollo de una actividad laboral y la dispensación de los debidos 
cuidados. Sin embargo, y pese a que la atención sociosanitaria constituye un elemento esencial en cualquier política de apoyo a las situaciones de dependencia, también es cierto que desborda el ámbito de ésta para proyectarse hacia otras situaciones que requieren la prestación combinada de medidas sanitarias y sociales.

- Una última aclaración gira en torno a la actual regulación de los Servicios Sociales en nuestro país. Actualmente, la Constitución Española ${ }^{4}$ reconoce la posibilidad de que las CC.AA., a través de sus respectivos Estatutos de Autonomía puedan asumir la competencia exclusiva en materia de "asistencia social", lo que en el caso de Andalucía tuvo su plasmación en el artículo 13 apartado 220 del Estatuto de Autonomía, y en su desarrollo legislativo posterior (Ley del Parlamento Andaluz 2/1988, de 4 de abril, de Servicios Sociales de Andalucía). Pues bien, dado que cada Comunidad ha organizado sus Servicios Sociales de forma autónoma, la regulación que se haga en el ámbito nacional del seguro de dependencia deberá o bien respetar el marco organizativo y normativo de cada Comunidad, lo que constituye una dificultad de orden esencialmente jurídico que deberá ser salvada a través de una compleja regulación estatal de mínimos, o bien modificarlo. Por otra parte, y centrándonos ahora en la Comunidad en la que residimos, la regulación autonómica andaluza de los Servicios Sociales no contempla las prestaciones propias de los Servicios Sociales (teleasistencia, plazas residenciales...) como un derecho subjetivo del individuo, lo que le permitiría exigir su prestación, en primer lugar, ante la Administración Pública competentey, en segundo lugar, ante cualquier Tribunal de J usticia. Muy al contrario, la regulación actual sólo prevé el derecho a recibir la prestación (por ejemplo el ingreso en una residencia asistida) para aquellas personas con respecto a las que, de acuerdo con un baremo objetivo, se determine que se encuentran en una situación de penuria y necesidad tal que resultan merecedores de especial atención. Esta situación puede estar próxima a cambiar, pero la financiación de los Servicios Sociales universales vuelve a ser una cuestión a tener en cuenta, sobre todo si dentro de las prestaciones a las que nos referimos englobamos la atención a personas dependientes.

La Comunidad Autónoma de Andalucía, al igual que el resto de CC.AA. (a excepción de Cataluña) aún no ha regulado la atención a la dependencia, y

4 Artículo 18.1.20 de la Constitución Española de 1978. 
hoy por hoy se guía por los criterios asistencialistas anteriormente descritos, sin perjuicio de que cada día sea mayor el número de recursos que la Administración autonómica, ya sea de modo directo o vía concierto, pone a disposición de los ciudadanos.

En nuestro país existen ya programas electorales, como es el caso del PSOE, que apuestan decididamente por establecer el derecho de cualquier ciudadano, por el mero hecho de serlo, a percibir del Estado una serie de prestaciones sociales, entre las que este partido incluye los supuestos de atención a la dependencia. Este partido político pretende alcanzar lo que se ha llamado el "cuarto pilar del esta do del Bienestar", la universalización de los servicios sociales públicos, lo que junto a la universalización de la sanidad, de la educación y de las pensiones constituyen los pilares de la sociedad que su proyecto político propugna.

Propuestas similares son esbozadas en los programas electorales de otras formaciones políticas como el PP y CiU, si bien es cierto que la universalización del derecho a los servicios sociales públicos, al igual que en su día la universalización del derecho a la educación o a la salud, conlleva consigo una partida presupuestaria tan importante que las propuestas suelen estar presididas por cierta prudencia política. En cualquier caso, la atención a la dependencia comienza a incorporarse a los discursos políticos con cierta normalidad, y el Estado, con independencia de la formación política que sustente al gobierno y del mayor o menor alcance de su protección, tendrá que dar respuesta a la situación de dependencia de sus ciudadanos.

Sin embargo, la universalización de los servicios sociales en lo que a la atención a las personas dependientes se refiere, no es una pretensión ni novedosa ni exclusiva de ninguna formación política, con independencia de que se muestre mayor o menor interés político en su implantación. Algún tiempo atrás, una Institución como el Consejo de Europa ya consideraba la cobertura del riesgo de la dependencia como una parte integrante del sistema global de protección social, e incluso iba más allá al afirmar que además de abarcar a la protección de las personas dependientes, debía alcanzar también a sus cuidadores.

\section{El seguro de dependencia: los modelos existentes y su financiación}

Una vez sentadas estas bases, conviene acercarse al seguro de dependencia analizando cuál fue su origen, y cómo ha sido regulado en distintos países. 
Un análisis comparado de las distintas regulaciones nos acercará más a la regulación que sobre esta figura pueda producirse en nuestro país.

El seguro de dependencia, como modalidad aseguradora, nace en EE.UU. a finales de los años cuarenta. No tuvo gran éxito en un principio, y su evolución fue lenta en sus comienzos, pero actualmente ofrece cobertura a decenas de millones de personas y alcanza unas cifras de negocio importantísimas.

La regulación de la asistencia sanitaria en EE.UU. es tan singular que merece ser reseñada brevemente, para así conocer la verdadera importancia de los seguros médicos en aquel país. En los EE.UU. existe un seguro público universal, llamado "Medicare", que cubre la asistencia sanitaria de todos los ciudadanos mayores de 65 años. Así mismo, con carácter voluntario, los ciudadanos contratan a lo largo de su vida seguros privados sanitarios que cubren una parte de la asistencia médica que puedan necesitar. En el caso de las personas menores de 65 años, si carecen de recursos económicos para contratar un seguro privado, la Administración dispone de otro programa Ilamando "Medicaid", que proporciona una mínima cobertura. No obstante, ni "Medicare" ni tampoco los seguros sanitarios privados tradicionales ofrecen cobertura para los gastos generados con motivo de la utilización de los cuidados formales (plazas residenciales) destinados a las personas dependientes. Este tipo de gastos son únicamente cubiertos por "Medicaid", para aquellos supuestos de auténtico desvalimiento social. En consecuencia, el ciudadano americano está obligado a prever de forma absolutamente privada durante su etapa de vida laboral, mediante cualquiera de los productos que el mercado le ofrece (seguros de invalidez, seguros de dependencia, planes de jubilación) cualquier eventualidad que le pueda acontecer durante su vejez.

Esta situación, cuando menos llamativa si se observa desde el punto de vista de los sistemas de protección social europeos (Seguridad Social española y francesa, por ejemplo), constituye, sin lugar a dudas, un perfecto caldo de cultivo para la proliferación de los seguros de dependencia y otros productos similares.

No fue hasta los años 80 cuando las compañías aseguradoras europeas empezaron a desarrollar tímidamente esta modalidad de seguro, si bien es cierto que como seguro estrictamente privado en ningún país europeo ha tenido éxito esta modalidad. 
Los aseguradores europeos siempre han mirado con recelo esta modalidad, dado que la cobertura que ofrece puede dilatarse mucho en el tiempo, haciendo escasamente rentable su actividad empresarial. Los americanos resolvieron esta cuestión estableciendo una muy severa prueba médica de acceso a esta modalidad de seguro, lo que evidentemente redunda en una menor siniestralidad, pero también reduce de forma importante el número de candidatos a acceder a esta modalidad de Seguro. De esta forma se entra en un círculo vicioso de difícil salida. No obstante, la regulación de la asistencia sanitaria y social en EE.UU. hace que, por necesidad, esta figura tienda a ser rentable, además de en términos sociales, en términos económicos.

En cualquier caso, la visión que sobre la dependencia se tiene en el continente europeo hace que, de forma casi natural, se tienda a incorporar esta prestación al Sistema Público de Protección Social. Nos encontramos ante un concepto, el de "Estado del Bienestar", que tiende a que los servicios esenciales de la comunidad se presten desde el sector público, aunque en absoluto se niega, e incluso se fomenta, la participación del sector privado como instrumento para lograr más recursos, y mejores servicios.

El país que primero reguló esta materia en Europa fue Alemania, contemplándolo como una prestación pública de carácter obligatorio que, dado que en este país rige un sistema de seguro sustitutivo o alternativo, puede ser mejorado mediante una cobertura adicional suscrita con una entidad aseguradora privada.

Conviene, por su interés, detenerse brevemente en el modelo alemán. En Alemania el gobierno ofrece una cobertura universal aunque limitada de las situaciones de dependencia, de tal forma que si un ciudadano quiere optar a recibir un mejor servicio, deberá suscribir una póliza de Seguro privada. El Estado Alemán sólo garantiza un nivel básico de atención que gradúa en tres niveles de dependencia en función de la frecuencia horaria con que se requieran los cuidados que la persona demande, y que a su vez, en función del grado de dependencia asignado por un equipo de profesionales formado por médicos y trabajadores sociales, conlleva aparejada una prestación asistencial a domicilio, una contribución económica o una combinación de ambas. La contribución económica es siempre igual para todos los ciudadanos alemanes que se encuentren en idéntica situación, con independencia de los recursos económicos de que dispongan. Con esta ayuda se entiende que el ciudadano puede recabar la prestación de la atención requerida, por 
ejemplo el ingreso en una residencia, con un nivel básico adecuado. De esta forma, si el ciudadano quiere recibir mayores y mejores prestaciones deberá acudir a la suscripción de un seguro privado de dependencia.

En Alemania, la financiación de la cobertura de la dependencia de sus ciudadanos se realiza mediante una cotización a la Seguridad Social del 1,7\% de los salarios brutos de los trabajadores, y por una aportación de los empresarios de igual importe. Curiosamente, en el proceso de negociación social tendente a la implantación de esta cobertura, empresarios y trabajadores acordaron reducir en un día las vacaciones de estos, con el fin de contribuir con la contribución de la producción de un día de la economía alemana al sostenimiento del sistema público de cobertura de la dependencia. Nos encontramos ante un interesante modelo de solidaridad intergeneracional con base en la concertación social.

El sistema alemán de seguro de dependencia, aprobado mediante una Ley del parlamento Federal de 1994, comenzó su aplicación el primero de enero de 1995, constituyendo la primera regulación legal europea de la materia.

En Alemania el sector privado actúa como complementario de la cobertura básica anteriormente referida que es ofrecida por el Estado Federal, y unos 8,5 millones de personas tienen suscritos seguros de dependencia con compañías privadas.

Bélgica fue el segundo país europeo que reguló el seguro de dependencia. Ocurrió en 1999, y lo hizo de forma muy similar al modelo alemán, aunque de forma más sencilla.

Por su parte, Francia es el mercado europeo más desarrollado para el seguro de dependencia, después de Alemania. En Francia hay suscritas 600.000 pólizas privadas de dependencia. En 1996 entró en vigor en este país una Ley de Cuidados a los Mayores, cuyos beneficiarios son las personas mayores de 60 años, y cuyas prestaciones están sujetas a la situación económica del mayor, e incluso condicionadas a una reserva sobre su herencia.

Luxemburgo ha sido el último país europeo en regular esta materia, y su regulación ha sido el resultado de un serio debate nacional. Al final, Luxemburgo adoptó el sistema de cobertura pública vía "contribución social obligatoria", que es como se denomina en este país lo que para los españoles es la cotización a la Seguridad Social, aunque ha introducido la modalidad de copago para suavizar la repercusión que la cobertura de las eventuales 
situaciones de dependencia de sus ciudadanos supondría para el presupuesto nacional.

Por hacer alguna referencia a la regulación internacional no europea, merece reseñarse, por su singularidad, el caso de Japón. En este país, el nuevo seguro social de dependencia comenzó a funcionar a mediados del año 2000, siguiendo una vía ligeramente distinta a la del modelo alemán. Las prestaciones que realiza el seguro japonés de dependencia son siempre en especie, satisfaciendo el Estado el $90 \%$ del precio del servicio que en cada caso elija el beneficiario, corriendo el 10\% restante a cargo de éste. La financiación del sistema corresponde a un sistema mixto; un $50 \%$ procede de las cotizaciones a la Seguridad Social de los ciudadanos mayores de 40 años de edad, y el $50 \%$ restante a los ingresos generados por el sistema impositivo general. Esta peculiaridad de cargar el peso de las situaciones de dependencia sobre las personas mayores de 40 años de edad tiene su justificación en que es precisamente a estas generaciones a las que correspondería hacerse cargo de sus familiares dependientes en el supuesto de que no lo hiciera el Estado. Puesto que se benefician de ello, deberán pagar en mayor proporción por la prestación recibida. Esta regulación constituye una singularidad mundial en la regulación del seguro de dependencia.

En Australia, la atención a largo plazo se proporciona por el sector privado a través de organizaciones especializadas, algunas de ellas benéficas y otras con ánimo de lucro.

Un interesante estudio ${ }^{5}$ de la Organización Mundial de la Salud (OMS) apuesta por novedosas formulas de asociación entre gobiernos y los aseguradores privados de salud. Se trata de una propuesta mixta que parece estar ganando apoyos, pues los recursos gubernamentales tienden a ser insuficientes ante el volumen de servicios que irá demandando la sociedad para atender a sus miembros dependientes. Quizás aún sea pronto para verlo, pero el envejecimiento demográfico conlleva inherente unos problemas de financiación de muy alto calado, y realizar una correcta planificación de los recursos que sin duda se demandarán parece ser la actitud más inteligente a adoptar.

En realidad, podría decirse que existen dos concepciones, casi antagónicas, en relación con la regulación del seguro de dependencia. Por un lado, y por orden de antigüedad, el modelo de los EE.UU, en el que se acude a una

5 OMS, (1999). 
modalidad de seguro estrictamente privado, mientras que por otro se encuentra el modelo de Alemania, Bélgica y J apón, que se basa en un sistema de seguro público, integrado en los sistemas de seguridad social, que prevé la colaboración del sector privado con carácter complementario (no sustitutivo). Se trataría, pues, de un modelo mixto.

\section{TABLA 1}

\section{Modelos de regulación del seguro de dependencia}

\begin{tabular}{|l|l|}
\hline Modelo EE.UU. & Seguro privado \\
\hline Modelo alemán, belga y japonés & $\begin{array}{l}\text { Seguro mixto; de carácter público, } \\
\text { pero que admite de la complemen- } \\
\text { tariedad de las pólizas privadas }\end{array}$ \\
\hline
\end{tabular}

Curiosamente, y dado el enorme coste que ello supondría para las arcas públicas, no existe ningún modelo estrictamente público de cobertura universal de la dependencia.

En los países europeos que aún no han legislado sobre esta materia es previsible que se acabe imponiendo el modelo mixto alemán.

En este punto estamos en condiciones de poder afirmar que la mayor parte de los escasos países que han regulado el seguro de dependencia hasta la fecha han optado por un esquema de aseguramiento público universal, pero ello no impide el que existan notables diferencias entre ellos en lo que respecta a la financiación de sus respectivos sistemas. Básicamente se podría decir que existen dos enfoques al respecto:

- Por un lado el de Alemania y Japón, donde el grueso de la financiación procede de las cotizaciones a la Seguridad Social,

- Y por otro, el de los países escandinavos, donde los recursos que financian el sistema público de cuidados de larga duración (long-term care), es decir, las situaciones de dependencia, proceden en su inmensa mayoría de los ingresos generados por el sistema impositivo en su conjunto.

Anteriormente hemos analizado brevemente el sistema de financiación de los sistemas alemán y japonés, y dada la relevancia que tienen las políticas 
sociales en los países escandinavos, consideramos necesario hacer una sucinta referencia sobre su regulación y sus fuentes de financiación. Dado que no existen sustanciales diferencias en su regulación, salvo en el caso de Dinamarca que posteriormente comentaremos, nos referiremos de forma global a Noruega, Suecia, Dinamarca y Finlandia.

A diferencia de Alemania y Japón, países en los que la financiación de la dependencia corre a cargo de las cotizaciones de empresarios y trabajadores a la Seguridad Social, en los países escandinavos es la sociedad en su conjunto la que, mediante el pago de los impuestos sostiene el sistema existente. En concreto, son tres las fuentes de financiación utilizadas:

- Por una parte, el Gobierno Central cede a los Gobiernos Subcentrales (condados, municipios...) parte de los ingresos obtenidos a través de los impuestos estrictamente estatales, sin que exista ningún impuesto específicamente destinado para esta cuestión.

- Por otra, los Gobiernos Subcentrales realizan idéntica operación, incrementando con sus recursos propios (impuestos locales) la asignación estatal.

- Y finalmente, la tercera fuente de financiación la constituyen los copagos; esto es, los usuarios pagan de su propio bolsillo una parte proporcional del coste de los servicios que utilicen, variando el porcentaje en función del tipo de servicio requerido.

En el caso de las residencias, la aportación que realiza el usuario viene determinada por la cuantía de su pensión. De hecho, resulta interesante saber que en los países escandinavos, a excepción de Dinamarca, la residencia ingresa directamente la totalidad de la pensión de los residentes, dejando a éste una pequeña cantidad en concepto de "dinero de bolsillo", que es idéntica para todos los ancianos. Por el contrario, En España es el residente quien recibe la totalidad de la asignación económica, debiendo aportar el $80 \%$ de ésta al centro residencial en el que se aloje, y manteniendo el $20 \%$ restante como "dinero de bolsillo". Sin embargo, y coincidiendo con el modelo español, en los países escandinavos es el Estado quien sufraga la diferencia de coste entre el precio del servicio y la aportación del usuario en los casos en los que la aportación del residente no alcance a cubrir el coste de su estancia.

Un interesante trabajo de investigación de la Unión Europea realizado en 1999, asegura que el subsidio público medio en Noruega, Suecia y Finlandia 
se sitúa en torno al $80 \%$ del coste de una plaza residencial en sus respectivos países. Lo que "a contrario sensu"indica que el pago medio que realiza el usuario es de un $20 \%$ del coste, lo que ofrece una clara idea del nivel de protección social de aquellos países.

El caso de Dinamarca es algo diferente. Aquí el usuario retiene completamente su pensión, pero debe aportar el $15 \%$ de to da su renta (pensión y otros ingresos) a la residencia, además de pagar una serie de tasas de cuantía fija por diversos conceptos (alojamiento, manutención, electricidad...). En este supuesto no existe el copago.

No obstante, y con carácter general, el sistema de universalización de los servicios públicos de atención a las personas dependientes en los países escandinavos es bastante más justo, en términos sociales, que el existente en Alemania. La razón es clara, los países escandinavos han introducido el copago como mecanismo corrector de las distintas situaciones económicas de los usuarios, haciéndose cargo el Estado de una mayor parte del coste de los servicios requeridos por las personas que disponen de menores recursos económicos. Por el contrario, el sistema establecido por el seguro alemán de dependencia asigna idéntica cantidad de dinero a todas las personas que padezcan igual grado de dependencia, con independencia de su nivel de ingresos.

TABLA 2

Vías de financiación de algunos sistemas de cobertura de la dependencia

\begin{tabular}{|l|l|}
\hline Sistema de EE.UU. & Pólizas privadas \\
\hline Sistema japonés & $\begin{array}{l}\text { - } 50 \% \text { cotizaciones a la Seguridad Social de los } \\
\text { mayores de } 50 \text { años } \\
\text { - } 50 \% \text { vía impuestas }\end{array}$ \\
\hline Sistema alemán & $\begin{array}{l}\text { - Cotizaciones a la Seguridad Social } \\
\text { Pólizas privadas para prestaciones } \\
\text { complementarias }\end{array}$ \\
\hline $\begin{array}{l}\text { Sistema de los países } \\
\text { escandinavos }\end{array}$ & - Exclusivamente impuestos \\
\hline
\end{tabular}


A pesar de que no hagamos expresa mención a ellos, los países que han optado por un sistema que la doctrina denomina "asistencialista", el copago constituye una importante fuente adicional de financiación de los servicios ofrecidos por la Administración. Así, para financiar el coste de las plazas residenciales, muchos países, entre ellos el Reino Unido, Irlanda, Portugal, Holanda, Austria o España, permiten que el usuario se quede con una pequeña parte de su pensión en concepto de "dinero de bolsillo", pero obligan a dedicar el resto a satisfacer el coste de su estancia. En el caso de que la cantidad resultante sea insuficiente, es el Estado el que se hace cargo de la diferencia.

Otros países como Bélgica, Italia o Francia ofrecen una ligera variación. En estos casos, el coste de una plaza en residencia se divide en dos componentes: el sanitario y el residencial. El primero (cuidados de enfermería, consumo farmacéutico...) lo cubre directamente el Estado a través de sus presupuestos en materia de Sanidad, y el segundo, el componente residencial, que es el de mayor importancia económica, lo paga el residente de su propio bolsillo, e incluye todos aquellos gastos no sanitarios (alojamiento, manutención...). En el caso de que el usuario no alcance a satisfacer su coste, la Administración asume la diferencia.

Una vez llegados a este punto, conviene apuntar como primera conclusión que frente a las tradicionales carencias existentes en lo que se refiere a la atención residencial y domiciliaria en los países del sur de Europa, (España, Italia, Portugal y Grecia), algunos de los países más avanzados de Europa ya han ido tomando conciencia del problema y aplicando inteligentes soluciones desde una perspectiva financiera, fiscal y de servicios.

Pues bien, una vez analizada la regulación internacional sobre las situaciones de dependencia, conviene centrarnos en cómo se plantea la regulación de la materia en nuestro país. Trataremos de dar respuesta a la siguiente pregunta:

\section{4. ¿Hacia qué modelo de Seguro de Dependencia se dirige España?}

En 1999, el ministerio español de Trabajo y Asuntos Sociales, en colaboración con la Universidad de Alcalá de Henares, publicó un interesante informe sobre el seguro de dependencia, Este informe, junto con el elaborado por Unespa constituyen las principales fuentes de información sobre la materia en nuestro país y, el primero de ellos, es el que nos permite conocer la 
propuesta de regulación sobre la materia que en un futuro próximo elevará el Gobierno español al Parlamento.

De hecho, la Ley de Presupuestos para 2000 (Ley 55/1999) en su disposición adicional 140 ya decía:

"El Gobierno, en el plazo de seis meses, presentará a las Cortes Generales un informe relativo al Seguro de Dependencia, con una propuesta de regulación, un marco fiscal que la incentive y las modificaciones normativas necesarias para que pueda ser una prestación realizada por los planes de pensiones, las Mutualidades de Previsión Social y demás Entidades Aseguradoras".

Lamentablemente, el plazo expiró sin que el citado informe se presentara a las Cortes Generales, y aún sigue sin haber recibido trámite parlamentario. No obstante, un informe de la Dirección General de Seguros, que habrá de servir de base y fundamento para el contenido de este informe, es claramente favorable a la creación de esta modalidad, y hace especial hincapié en la conveniencia de que en su regulación se contemplen amplios beneficios fiscales.

El Gobierno se ha comprometido a presentar el Proyecto de Ley del Seguro de Dependencia a las Cortes Generales antes de que concluya la actual legislatura. Habrá que estar atentos a su contenido para conocer la modalidad de Seguro por la que apuesta nuestro país, aunque todo parece apuntar a que se trate de un seguro de tipo mixto basado en el modelo alemán.

Ante la situación actual, el sector privado sin duda puede ofertar pólizas de seguros que cubran los supuestos de dependencia, pero ante la proximidad de su regulación estatal, la aún escasa conciencia social sobre la materia y las expectativas de disfrute deimportantes beneficios fiscales en las pólizas de nueva suscripción, un elemental sentido de la cautela está haciendo que el sector se prepare, pero no oferte productos deestas características, si bien existen interesantes excepciones ${ }^{6}$.

Baste citar, a modo de simple curiosidad, que según estudios realizados por la compañía aseguradora Sanitas las primas anuales que habrán de satisfacer los beneficiarios del seguro privado de dependencia en España

6 "Horizonte Generali", producto de la compañía Estrella Seguros, garantiza una suma anual y vitalicia entre 3.005 y $18.030 €$ ( 500.000 y 3.000 .000 ptas.) para pago de servicios, dependiendo del grado de dependencia asignado. El seguro de dependencia de Centro Asegurador garantiza el $80 \%$ de los gastos derivados de la situación de dependencia. Sanitas Dependencia, de la compañía aseguradora Sanitas, garantiza una ayuda económica de hasta $1.502 €$ mensuales (250.000 ptas.) para prestación de servicios. 
oscilarán en torno a las siguientes cifras: para una persona de 40 años de edad: $2.190 €(364.500$ ptas.), de 50 años: $2.560 €(425.900$ ptas.), de 60 años: $3.123 €(519.600$ ptas.), y de 70 años: $3.615 €$ (601.600 ptas.).

Como se ve, la cifra tiene la suficiente entidad como para sopesar mucho la necesidad de sumarla a los múltiples gastos fijos ya soportados por el ciudadano medio. Huelga comentar que la cuantía de la prima se encuentra en relación directa con el éxito de cualquier modalidad de seguro.

Pues bien, llegados a este punto, y a la vista del análisis comparado de la regulación europea y extracomunitaria anteriormente reseñada, considero que es el momento adecuado para verter una opinión acerca del modelo que considero sería el idóneo para ser adoptado por nuestro país. Desdemi punto de vista, nuestro país debería optar por un modelo de seguro de dependencia público y mixto, similar al alemán, en el que se garantice la universalidad de una cobertura básica, pero no se excluya la complementariedad de las pólizas privadas y en el que, a mi juicio, deberían introducirse las medidas correctoras contempladas por los países escandinavos, a excepción de Dinamarca, e importantes beneficios fiscales que hagan atractivo la contratación de pólizas privadas complementarias.

\section{Análisis de las características esenciales del seguro de dependencia}

Aunque de manera sucinta, pues pretendemos realizar nuestra aproximación a esta modalidad de seguro desde un punto de vista eminentemente social, y no jurídico-mercantil, si queremos analizar con cierto rigor el seguro de dependencia debemos adentrarnos en algunas de sus peculiaridades.

- En primer lugar analizaremos su cobertura. ¿Ante qué situaciones deberá responder el seguro? ¿Quién determinará si una persona es dependiente o no? ¿En qué medida le afecta su dependencia para las actividades básicas de la vida diaria (ABVD)? ¿Variará la contraprestación del Seguro en función del grado de dependencia del asegurado?

- Un segundo aspecto a tener en cuenta es la fiscalidad de esta figura. Si como todo parece indicar, la regulación que realice nuestro país no contempla el carácter obligatorio de esta modalidad de seguro y, por otra parte, no se concediesen importantes incentivos fiscales para su suscripción, serían pocos los ciudadanos que se decidiesen a hacerlo, lo que sin duda, a largo plazo, acabaría propiciando el fracaso de esta modalidad de seguro y el consiguiente cuestionamiento de la solvencia 
del sistema público de Seguridad Social, que sería incapaz de soportar por sí solo tal demanda de servicios.

- Por último, habrá que analizar aspectos estrictamente jurídico-mercantiles de esta modalidad de seguro, pero con mucha trascendencia social, como son el importe de la póliza, el periodo de carencia, la edadlímite de contratación y la opción entre pago en metálico o la prestación del servicio.

\subsection{Cobertura del seguro de dependencia}

Ante una situación de dependencia, la primera tarea es clasificarla según su gravedad.

Si recordamos la definición anteriormente dada a la dependencia, recordaremos que se trata de una situación que priva a un individuo de manera definitiva de la autono mía personal necesaria para el desarrollo independiente de las actividades básicas de la vida diaria (ABVD), necesitando dela ayuda o supervisión de otra persona para la realización de las mismas. Pues bien, como primer paso conviene determinar de forma clara cuáles son estas actividades cuya realización se ve seriamente condicionada. La OMS ha reconocido que los parámetros fundamentales para determinar la dependencia de una persona, o sea, las ABVD que deben verse afectadas, son:

- El vestido.

- La alimentación.

- La continencia.

- El aseo.

- El desplazamiento.

- La higiene personal.

Atendiendo a estos parámetros, se han creado diversas tablas de valoración funcional que vienen a atribuir un índice de dependencia a cada persona en función de los resultados obtenidos en pruebas fácilmente mensurables.

Entre las tablas de valoración funcional más conocidas se encuentran el Índice de Barthel y el Test de Medida de la Independencia Funcional (MIF), entre otros, como pueden ser las escalas de valoración de la dependencia de la Cruz Roja, o el Índice de Katz. 
- El Índice de Barthel consiste en la medición de la valoración física del individuo, mostrando resultados bajos para las personas dependientes y altos para las no dependientes.

- El test de Medida de la Independencia Funcional (MIF) es un indicador básico de las deficiencias y discapacidades, siendo muy sensible a pequeñas variaciones.

Pues bien, combinando los resultados obtenidos en varias tablas de valoración funcional, se puede lograr una graduación del nivel de dependencia en tres niveles:

- Grado I de dependencia, dependencia leve o dependencia baja: es aquella situación de dependencia en la que se encuentran afectadas 3 - 4 ABVD, con resultado del índice de Barthel entre 80 y 60 , y resultados de MIF entre 100 y 80.

- Grado II de dependencia, dependencia moderada o dependencia intermedia, que es aquella situación de dependencia en la que se encuentran afectadas 4 o 5 ABVD, con resultados de índice de Barthel entre 60 y 40 , y MIF de 60-18.

- Grado III de dependencia, dependencia grave o dependencia elevada, que es aquella situación en la que se encuentran afectadas 5 o 6 ABVD, con resultados de Índice de Barthel entre 40 y 0, y de MIF, de 60 a 18.

Huelga comentar que la medición del grado de dependencia debe repetirse periódicamente dado que la situación de las personas dependientes evoluciona con el tiempo, normal y desgraciadamente, a peor.

En cualquier caso, en función del grado de dependencia del individuo, así será la prestación que deberá ofrecer el Seguro, y que podrá ser económica, de servicios o mixta, pudiendo ir desde el apoyo en el domicilio del asegurado, hasta el ingreso en una residencia de asistidos.

Del mismo modo, resulta de justicia para mantener el principio de equilibrio contractual que, en el caso de que la prestación del seguro consista en la entrega de una cantidad de dinero, ésta varíe porcentualmente en función del grado de dependencia asignado. (Por ejemplo: 30\% para dependencia leve, $75 \%$ para dependencia moderada, y $100 \%$ para dependencia grave).

Tanto la determinación del grado de dependencia como la prestación que determine como procedente la compañía de Seguros, serán un evidente foco de conflictos con los asegurados, y de su pronta solución dependerá la 
atención recibida por la persona dependiente. (Ej. Mantener cuidadores en el hogar o instar el ingreso en una Residencia. El coste de estas medidas es sustancialmente distinto y su mantenimiento prolongado en el tiempo supone un claro perjuicio para la compañía, pero ¿y para el asegurado? ¿Qué coste personal supone el abandono del hogar? ¿Quién deberá determinar cuándo esta medida, y no otra, es la correcta?).

En este sentido, se han formulado interesantes propuestas por parte de las propias compañías de Seguros, las cuales han llegado a sugerir la creación de Órganos unitarios de valoración de la dependencia, de tal forma que todas las compañías acaten el criterio técnico emitido por un equipo multidisciplinar de profesionales. Yendo aún más allá, y previendo las eventuales situaciones de conflicto que habrían de generarse, al gunas de las regulaciones del seguro de dependencia existentes han contemplado la creación de un Tribunal facultativo público que determine la medida más correcta a aplicar en estos casos.

La figura del seguro de dependencia podrá gozar de una excelente regulación técnica, pero el principal problema consiste en dotarse de instalaciones de calidad en número suficiente para atender a un colectivo humano tan importante en términos cuantitativos. Al día de hoy es evidente que no existe en nuestro país una infraestructura suficiente para ofrecer cobertura a toda la población dependiente, pero no lo es menos el hecho de que si esta modalidad de seguro resulta de interés para el ciudadano, el sector privado, manejando un multimillonario fondo constituido por todos los depósitos de los asegurados, pronto creará la infraestructura material necesaria para la prestación de los servicios que sean requeridos. En esto precisamente radica el éxito de esta cobertura, crear una infraestructura de calidad con el menor gasto público posible, apoyándose en el Sector del Seguro Privado.

Un asunto de auténtico interés en el cuidado de las personas dependientes es el de la asistencia informal, lo que socialmente se conoce por la atención ofrecida por el "cuidador informal". Resulta indudable que la humanidad del trato ofrecido por el cuidador informal ${ }^{7}$ es difícilmente superable en un entorno institucionalizado. Siendo conscientes de ello, así como del bajo coste que pudiera suponer el pago de estos servicios, las propias compañías aseguradoras han optado por requerir del Gobierno que regule el desempeño de esta prestación de servicios similares a los de enfermería o a los de un

7 En España, el perfil del cuidador informal es el de una mujer, familiar del dependiente y con una edad media de 52 años. 
auxiliar de clínica y que son realizados por personas no tituladas, de forma que se establezcan los límites y la licitud de su actuación. De hecho, ya se está hablando de la creación de un Registro de Cuidadores Informales, con la finalidad de que, con los debidos controles, se les pueda ofrecer una asignación económica por parte de las compañías que deban ofrecer cobertura a la situación de dependencia de la persona a su cuidado.

Los cuidadores informales en nuestro país son merecedores del mayor de los reconocimientos sociales, y asumen una carga pesada e ingrata que con demasiada frecuencia constituye una fuente de no pocos conflictos personales y familiares. Una clara muestra de la importancia social de este colectivo la constituye el hecho de que el $73 \%$ de la población española dependiente es atendida por cuidadores informales. Baste este dato para valorar no sólo la importancia de su labor, sino el hecho de que sobre estas familias recae, de forma silenciosa, casi las tres cuartas partes del coste de la atención a las personas dependientes de nuestro país.

Otra cuestión de interés en el Sector del Seguro lo constituye la selección de riesgos que toda compañía aseguradora debe realizar para calcular el índice de siniestralidad de la incidencia a la que pretende ofrecer cobertura. Se trata, pues, de determinar qué riesgos deben de quedar excluidos, cuáles deberán quedar cubiertos y cuáles no serán ni siquiera asegurables. Esta es la pieza clave de la regulación norteamericana del seguro de dependencia. En EE.UU. se han establecido unos criterios muy severos para determinar el perfil aceptable de beneficiario del seguro de dependencia, lo que conlleva una baja siniestralidad y un mayor beneficio económico de las compañías aseguradoras. Las compañías de Seguros deben realizar un estudio de las patologías que favorecen la dependencia (Alzheimer, Parkinson, demencias, esclerosis múltiple, etc.), determinar las que son susceptibles de cobertura, establecer las prestaciones que podrán ofrecerse y, sólo entonces, podrán fijar la duración media y el coste medio de la situación de dependencia, lo que les permitirá establecer las primeras tarifas de riesgo, y por ende, las primas del seguro.

Hasta ahora, al tratar la cobertura del seguro de dependencia hemos manejado lo que podrían llamarse unos presupuestos sanitarios de la dependencia (tablas de valoración funcional, Órganos de valoración de la dependencia., asistencia informal, selección de riesgos). La clara fijación de estos parámetros es absolutamente fundamental para la regulación jurídicotécnica del seguro de dependencia. Sin ellos nos moveríamos en el mundo de 
las ideas. En términos jurídicos, lo que se está dilucidando no es otra cosa que el objeto del contrato.

\subsection{Fiscalidad}

Intentaré ser breve en este punto, pues lo verdaderamente interesante es que la regulación de esta modalidad de seguro ofrezca un tratamiento fiscal favorable que incentive su contratación. El interés social, y la paralela y sustancial reducción de costes para la Sanidad Pública de este producto, lo justifican. En esto parecen coincidir tanto la Patronal del Seguro (Unespa) como la propia Dirección General de Seguros del Ministerio de Economía.

Las demandas del Sector Asegurador en materia fiscal son las siguientes:

1. Deducciones sobre la cuota del Impuesto sobre la Renta de las Personas Físicas (IRPF) equivalentes a la mitad de la que disfrutan los Planes de Pensiones, o lo que es lo mismo, a $3.606 € / a n ̃ o ~(600.000$ ptas./año).

2. Exención de tributación como renta del trabajo en el IRPF de las cantidades que se paguen a familiares y a personal informal que cuide a las personas dependientes, hasta el límite del importe del Salario Mínimo Interprofesional (SMI).

3. Exención fiscal de las prestaciones económicas que puedan abonarse.

4. Establecimiento de un tipo muy reducido de IVA para las prestaciones sujetas a este impuesto.

Un favorable tratamiento fiscal de esta modalidad de seguro constituye el mejor incentivo posible para que los ciudadanos se decidan por invertir el dinero suficiente para cubrir unos servicios cuyo coste es a medio plazo difícilmente asumible por el sistema público de protección social.

\subsection{Aspectos jurídico-mercantiles}

- El período de carencia

Esta modalidad de seguro requiere la determinación de la edad a partir de la cual se podrá demandar la cobertura de la situación de dependencia, con independencia de la fecha de suscripción de la póliza. En este punto el Sector 
Asegurador parece coincidir en la edad de 65 años.

No obstante, se plantea la posibilidad de establecer un supuesto excepcional y anticipar la cobertura para los supuestos de dependencia sobrevenida por causa accidental. Esta medida parece razonable y su incidencia debe ser mínima en la determinación de la prima a satisfacer por el asegurado.

Un segundo supuesto de carencia lo constituye el período que habrá de transcurrir desde la notificación de la existencia de una situación de dependencia de un asegurado, hasta el momento en el que el seguro comienza a ofrecer la prestación que se determine. Se trata del tiempo necesario para determinar el grado de dependencia, el establecimiento del esquema de servicios adecuados al caso y, en definitiva, para organizar la prestación del servicio. Con respecto a este período, las compañías pretenden extenderlo al máximo en el tiempo para así reducir los costes a los que deberán hacer frente. De esta forma, sugieren su fijación en un período que puede oscilar entre los 30 y los 90 días.

\section{- Edad límite de contratación}

La fijación de una edad máxima de contratación muy avanzada conllevaría el establecimiento de unas primas muy elevadas, de esta forma todo parece indicar que la edad máxima de contratación podría fijarse en torno a los 55 años, con un perío do de carencia antes de tener derecho a la prestación de no menos de diez.

Esta ha sido la opción de los aseguradores americanos, y a priori parece razonable.

\section{- Riesgos asegurables}

A mayor rigurosidad en el reconocimiento médico, menor será el número de situaciones de dependencia susceptibles de cobertura, y por ende el coste de su asistencia, lo que permitirá rebajar las primas. Por el contrario, la compañía que sea más generosa en la admisión de riesgos deberá encarecer la prima de to dos los asegurados. En este sentido, la compañía nunca pierde, pero la cobertura ofrecida al asegurado puede ser muy diferente.

En cualquier caso, si se optase por un supuesto de colaboración entre el sector público y el privado, el llamado "modelo mixto", se tenderá a la 
socialización del riesgo, quedando muy en segundo plano los criterios de selección del riesgo, distribuyéndose éste de forma solidaria entre la "comunidad de asegurados", y asumiendo su coste entre todos ellos.

- Relación entidad aseguradora-asegurado dependiente

El principal foco de conflicto en esta modalidad de seguro se encuentra en las situaciones de discrepancia en la determinación del grado de dependencia del beneficiario y en la fijación del Plan Asistencial que se le establezca. Este aspecto ha sido comentado anteriormente, y resulta especialmente delicado si somos conscientes que una de las partes contratantes se encuentra en una situación claramente desigual, en una situación de dependencia.

En EE.UU. este tema se ha resuelto mediante sistemas de arbitraje especiales que ofrecen al asegurado una asistencia técnica.

En Alemania, se ha creado un organismo público que tiene encomendada la misión de valorar la dependencia y determinar el tratamiento a aplicar, resolviendo de forma sumaria cuantos conflictos puedan surgir.

En España, se comienza a hablar de la posibilidad de crear un servicio común y único para todas las entidades aseguradoras, técnico e imparcial, que determine el grado de dependencia y el plan asistencial adecuado a cada caso.

\section{- Primas}

La prima a satisfacer por el asegurado podrá ser única o periódica.

Probablemente deberá ser a fondo perdido, salvo situaciones excepcionales (Ej.: fallecimiento de asegurado de 63 años de edad que suscribió la póliza con 50 años, no iniciándose la posible prestación hasta los 65).

Lógicamente no podrá contratarse el seguro cuando la persona ya se encuentre en situación de dependencia. (Sería un supuesto de contrato nulo, puesto que el riesgo a cubrir ya ha acontecido).

- Naturaleza técnica del Seguro

Se trata de un sistema de capitalización, que sólo se convierte en renta vitalicia, pago único del capital acumulado o un derecho a la prestación de 
un servicio, una vez que se alcanza una determinada edad o se produce la situación de dependencia.

- Protección del asegurado

La situación de dependencia del asegurado es merecedora de especial atención, dado que es posible que a la hora de reclamar el servicio no se encuentre en pleno uso de sus facultades mentales, y en consecuencia de defender sus derechos.

Nos encontramos ante un dilema ético que debe ser resuelto por una entidad ajena a los intereses en conflicto.

Las aseguradoras americanas han resuelto la cuestión fijando en la misma póliza la figura de un tutor o curador, muy frecuentemente despachos profesionales, que vele por los intereses de su cliente y a los que se les deberá cumplida cuenta de todas las cuestiones que afecten al asegurado, y que podrán intervenir en su beneficio.

Un elemental principio de justicia nos indica que la determinación de los servicios a ofrecer no puede quedar al arbitrio de la única parte contractual que estará en condiciones de hacerlo. (ej.determinación de cuándo y por cuánto tiempo se van a mantener los cuidados en el entorno habitual de vida de un paciente terminal, y cuándo se habrá de realizar su traslado a un centro residencial o socio-sanitario).

Por otra parte, la compañía habrá de realizar una labor de protección del asegurado, de tal forma que se garantice que las prestaciones que ofrece van en su beneficio personal, y no en el de su entorno familiar más cercano, o en el de terceras personas.

\section{Conclusión}

El progresivo envejecimiento de la población conlleva el surgimiento de problemas ante los que la humanidad nunca hasta ahora ha tenido que enfrentarse. Esto, lejos de constituir un serio inconveniente, debiera servir de acicate para que nuestra sociedad se prepare para ofrecer respuestas a las nuevas necesidades emergentes. La atención a las situaciones de dependencia de nuestros mayores es un buen ejemplo de ello, y la principal cuestión se centra en torno a la financiación del gasto que ello conlleva. 
España está a punto de regular la atención de las situaciones de dependencia de sus ciudadanos, y es este el momento de observar con detenimiento las soluciones que al mismo problema han dado los países de nuestro entorno. Todo parece indicar que nuestro país optará por un modelo mixto, inspirado en el modelo alemán, con una importante cartera de servicios prestados a través de la Seguridad Social y que admitirá la complementariedad de los seguros privados, a los que se primará mediante importantes deducciones sobre la cuota del IRPF.

En cualquier caso, y salvo que se optara por la cobertura estrictamente privada de los supuestos de dependencia, cosa improbable, la universalización de su cobertura pública conllevará un sustancial incremento del gasto social que habrá que financiar ya sea por la ciudadanía en general a través de impuestos, o por los sectores productivos del país (empresarios y trabajadores) a través de cotizaciones a la Seguridad Social. Sin embargo, y tratando de no resultar alarmista, no debemos olvidar que en los países en los que está vigente esta modalidad de seguro, el gasto de protección social es mucho más elevado que en España. En nuestro país el gasto social representa el $24,5 \%$ del PIB, mientras que en Alemania supone el $31 \%$ y en Holanda el $33,6 \%$ pero, ¿estamos dispuestos los españoles a incrementar nuestra aportación al Estado o a suscribir una póliza de seguro para financiar la cobertura de una eventual situación de dependencia? Todo parece indicar que sólo aquellos ciudadanos que actualmente hacen frente a una carga, tanto económica como emocional y familiar, como ésta son conscientes de la gravedad de la cuestión. Pero con independencia de la escasa conciencia social existente sobre esta desconocida materia, no es menos cierto que nos enfrentamos a una cuestión de solidaridad intergeneracional de la que muchos de nosotros podremos beneficiarnos en un futuro. Recordemos a mero título de ejemplo que la mitad de la generación de los españoles nacidos en la década de los 60, en el Ilamado "baby-boom", tendrá la dicha, por primera vez en la historia de nuestro país, de alcanzar los 80 años de edad, y una parte significativa de este enorme grupo de población llegará a ser centenario. No es descabellado, pues, pensar que la probabilidad de padecer una situación de dependencia asociada al envejecimiento es más elevada de lo que a primera vista intuye el ciudadano medio español.

De cualquier forma, ante este mismo dilema, que esconde tras de sí un profundo contenido ético, se encuentran los países más desarrollados de nuestro mundo, entre los que tenemos la fortuna de encontrarnos. En su solución puede estar el futuro de muchos de nosotros, y sólo siendo cons- 
cientes de ello estaremos en condiciones de adoptar la mejor de las soluciones posibles.

\section{Bibliografía}

Belando Montoro, M. R., (2000), Educación y vejez social. Ámbitos y propuestas de intervención, Barcelona, Ed. PPU (Promociones y Publicaciones Universitarias)

Casado Marín, D. y López I Casasnovas, G., (2001), Vejez, dependencia y cuidados de larga duración. Situación actual y perspectivas de futuro, Fundación “La Caixa".

Comisión Europea, (1999), Informe de 21 de mayo de 1999. Hacia una Europa para todas las edades.

Comisión Europea, (2000), Comunicación sobre Tendencias sociales: perspectivas y retos, Com 2000/82.

Guerrero de Castro, M., (2001), "El Seguro de Dependencia”, Revista Española de Seguros, № 105, enero-marzo.

Montiel Torres, A. M., (1997), "Calidad de vida y tercera edad: algunos indicadores", Revista de Fomento Social, no 206, abril-junio, pp. 215-236.

OMS, (1999), World Health Report, 1998.

Parro Fernández, I., (1992). "Sociedades ancianas, sociedades de las solidaridades", Revista de Fomento Social, no 225, enero-marzo, pp. 115-132.

Pérez Díaz, J ., (2000), “Envejecimiento poblacional y dependencia: una perspectiva desde la necesidad de cuidados", Pamplona. (II J ornada Gerontológica. Recursos asistenciales, octubre 2000). 